\title{
ARTIFICIAL INTELLIGENCE IN OPTIMAL RePartition OF THE Reactive PoWer
}

\author{
Rogobete, M. \& Dumitrascu, M.
}

Abstract: Voltage and reactive power control is a major problem in the operation of energy systems, due to differences between the topology of transmission and distribution systems. Sequentially, a high interest is attributed to the ability to determine the optimal amount of energy that must flow through certain sections of the network to ensure the power quality and security in the delivery of energy to the consumer. As a power system has more consumers, it will include more generators, this process increasing the problem complexity of maintaining the voltage at the network nodes in the desired limits. Since reactive power cannot be transported over long distances, voltage control must be performed using special devices located at different positions of the system. This paper aims to find a viable alternative in order to select and command the devices for reactive power and voltage control.To a problem of such complexity, the optimal solution, or at least one acceptable, is difficult to achieve using traditional techniques. The reasons are related to the application of restrictive methods, but also due to laborious calculations required, translated into large amount of computation time. Using evolutionary computation - EC laws, inspired by natural evolution of species, is an alternative for solving such problems. This paper contains an original calculation, based on differential evolutionary algorithms to optimize the reactive power dispatch in a power system. Applying algorithms make an important contribution to improving methods of optimizing the distribution of energy in the studied systems.

Keywords: Artificial intelligence, power control, voltage control, optimal algorithm
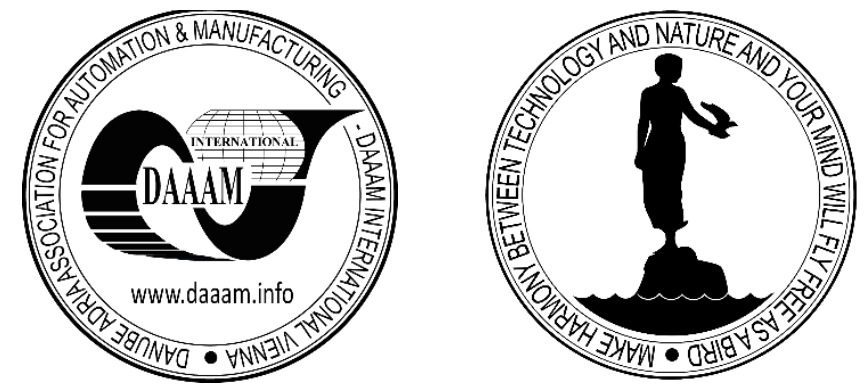

Authors' data: PhD. Eng. Rogobete, M[arius]*; Eng. Dumitrascu, M[arian]*, *GE Alstom Alliance Romania, 03254, Bucharest, Romania, marius.rogobete@gmx.de, dumitrascu_marian_0906@yahoo.com

This Publication has to be referred as: Rogobete, M[arius] \& Dumitrascu, M[arian] (2016). Artificial Intelligence in Optimal Repartition of the Reactive Power, Chapter 12 in DAAAM International Scientific Book 2016, pp.121-134, B. Katalinic (Ed.), Published by DAAAM International, ISBN 978-3-902734-09-9, ISSN 1726-9687, Vienna, Austria

DOI:10.2507/daaam.scibook.2016.12 


\section{Introduction}

The interest for controlling power flow possibilities in transmission systems has increased significantly over the past years. The reasons are coming from both the applications, represented the energy management system, but also on the technological side - the emergence of new system components such as semiconductor based devices. In many countries, operation of energy systems has changed due the increasing of the transmission network and the liberalization of the electricity market (Herbig \& Andersson, 1997).

In certain nonregulated environments, the transport capacity becomes a commodity because, to use the transmission capacity, a tax must be paid and the transmission system operator depends economically of these fees. In this reason it needs to be able to control the energy transmission as well as the high interest components. The new components are feasible if they are based on advances in power electronics and related technologies.

There are four main reasons for controlling power flow is attractive:

a. Mitigating overload.

In a supply system as a network, it can happen that a low impedance line to carry far more power than the original designed capacity, while parallel sections are under utilized. By controlling power flow line can be facilitated, resulting in an overall better use of the network.

b. Contractual requirements.

They usually come in either of two situations described above. Transfer of power in a network is likely to be accompanied by requests from neighboring utilities, which requires compliance with certain quality parameters of electricity, so that certain parts of their networks are not affected in a negative way.

c. The presence of individual projects for power transmission.

The idea is that private companies operate transmission lines and sell capacity or energy transport by stakeholders. In this environment, control power flow will have, obviously, to be controlled.

d. Reducing losses.

By controlling power flow, we can avoid over-loading / under-loading some subsystem areas, thus maximizing the efficiency of the energy transport, thus reducing losses (Bakare, 2001).

However, reduction of losses in a particular area of the system through optimal allocation of reactive power is a problem difficult to resolve due to the complexity of energy systems and their dynamic nature.

The body of manuscript begins with Introduction (1st page) at the top of and ends with the last reference at the bottom of last page of manuscript.

\section{General Presentation}

Although reactive power is required to operate more electrical devices, it can cause harmful effects on them as well as to the electrical infrastructure. Since the current flowing through the electrical system is greater than demand, excess power is 
dissipated as heat while moving reactive current resistive components such as wires, switches and transformers. Whenever energy is consumed, it is counted. There is no difference if the energy is consumed as heat energy or useful. We can determine how much reactive power using electrical devices by measuring their power factor, i.e. the ratio of active power and apparent power.

Power Factor 1 (i.e. 100\%), ideally it means that all electricity is applied in the direction useful energy. Typically, homes have global power factors in the range of from $70 \%$ to $85 \%$, depending on the type of working apparatus. Newer homes with the latest energy efficient appliances may have a general power factor of $90 \%$. Residential power meter reads only active power, i.e. which would have a power factor of $100 \%$. While most companies do not perceive directly electricity costs for reactive power in the case of residential consumers, it is a misconception to say that there is no economic benefit in power factor correction. Losses that occur in the network, due to the lack of the power factor correction, will be covered by the price increase.

\section{Theoretical Bases}

During everyday operation, energy systems can present both over-voltage and under-voltage. By controlling the production, absorption and reactive power flow at all levels of the system, it can be maintained within acceptable voltage profile and reduce transmission losses.

3.1 Reactive power control in power systems

Reactive power is controlled by voltage control devices, strategically located in order to absorb or generate reactive power. These compensators are used to obtain the desired reactive power, the power factor correction, voltage levels and the reduction of power losses.

The devices and methods mainly used for reactive power compensation are (Kundur, 1993):

- synchronous generators.

- synchronous condensers.

- FACTS devices.

- Change of plots position in transformers.

- Shunt devices.

- Switching transmission lines.

3.2 Power Flow Calculation. General Description. Classification. Models

A power system consists of all electrical installations intended to convert primary energy in electricity, transport it to areas of consumption and to distribute it to consumers, within the quality parameters (frequency, voltage). Due to the fact that electricity cannot be stored in large quantities, a close control of the energy parameters must be kept so that it can meet the following basic requirements:

- Coverage of the consumption curve.

- Satisfying sufficient power quality at certain levels of voltage, frequency while maintaining reliability in supply.

- Supply electricity at a lower cost and with as minimal impact on the environment. 
In economic terms, the power flow calculation helps minimize the transportation and distribution costs by determining the sectors where congestions will occur or even when the maximum permissible load is exceeded, followed by a disconnection of the line / lines.

The steps in determining the power flow solution are:

- Establishment of one-line diagrams and initial data.

- Modelling system elements:

- The calculation of the parameters for power lines and determining equivalent schemes.

- Modelling transformers and autotransformers.

- Representing the equivalent scheme for determining the power flow solution.

- Nodal admittance matrix formulation.

- Solving the model using the Newton-Raphson method.

\subsection{Newton-Raphson method}

Newton-Raphson method is a powerful technique which contains first degree derivatives when calculating new nodal voltage. Normally it takes just 3 to 5 iterations to solve the power flow problem regardless of system size. It is one of the most commonly used techniques for determining the power flow solution.

Newton-Raphson procedure is based on the Taylor series, truncated to the first derivative, as follows:

$$
f(x+\Delta x) \approx f(x)+\left.\frac{\partial(x)}{\partial x}\right|_{x}(\Delta x)
$$

Where $f(x+\Delta x)$ represents a linear approximation.

The update matrix equation is symbolically written in a mixed polar-rectangular form, as follows:

$$
\left[\begin{array}{c}
P_{1 \text { spec }}-P_{1 \text { calc }}^{(m)} \\
P_{2 \text { spec }}-P_{2 \text { calc }}^{(m)} \\
\vdots \\
P_{\text {Nspec }}-P_{\text {Ncalc }}^{(m)} \\
--- \\
Q_{1 \text { spec }}-Q_{1 \text { calc }}^{(m)} \\
Q_{2 \text { spec }}-Q_{2 \text { calc }}^{(m)} \\
\vdots \\
Q_{\text {Nspec }}-Q_{\text {Ncalc }}^{(m)}
\end{array}\right]=\left[\begin{array}{cc}
J_{1}=\frac{\partial P}{\partial \delta} & J_{2}=\frac{\partial P}{\partial|V|} \\
J_{3}=\frac{\partial Q}{\partial \delta} & J_{4}=\frac{\partial Q}{\partial|V|}
\end{array}\right]\left[\begin{array}{c}
\left(\delta_{1}\right)^{(m+1)}-\left(\delta_{1}\right)^{(m)} \\
\left(\delta_{2}\right)^{(m+1)}-\left(\delta_{2}\right)^{(m)} \\
\vdots \\
\left(\delta_{N}\right)^{(m+1)}-\left(\delta_{N}\right)^{(m)} \\
--- \\
\left|V_{1}\right|^{(m+1)}-\left|V_{1}\right|^{(m)} \\
\left|V_{2}\right|^{(m+1)}-\left|V_{2}\right|^{(m)} \\
\vdots \\
\left|V_{N}\right|^{(m+1)}-\left|V_{N}\right|^{(m)}
\end{array}\right]
$$

Where:

$P_{i \text { spec }}$ - Active power specified for the $i$ node

$P_{i}$ calc $^{(m)}$ - Active power calculated for the $i$ node at the $m$ iteration 
$Q_{i \text { spec }}$ - Reactive power specified for the $i$ node

$Q_{i \text { calc }}{ }^{(m)}$ - Reactive power calculated for the $i$ node at the $m$ iteration

$J_{i}$ - Jacobian matrix terms

$\delta_{i}{ }^{(m)}, \delta_{i}{ }^{(m+1)}-$ Voltage angle calculated for the $i$ node at $m$ and $m+1$ iteration

$\left|V_{i}\right|^{(m)},\left|V_{i}\right|^{(m+1)}$ - Voltage calculated for the $i$ node at $m$ and $m+1$ iteration

Or in abbreviated form:

$$
\left[\begin{array}{l}
\Delta P \\
\Delta Q
\end{array}\right]=\left[\begin{array}{ll}
J_{1} & J_{2} \\
J_{3} & J_{4}
\end{array}\right]\left[\begin{array}{c}
\Delta \delta \\
\Delta|V|
\end{array}\right]
$$

\section{State of the art. Optimal reactive power dispatch (ORPD).}

\subsection{Classical techniques}

The following methods belong to the classical techniques group:

- Linear programming.

- Nonlinear programming.

- Gradient method.

- Mixed integer programming.

- Interior points method.

- Sequential quadratic programming.

Grudini (Grudinin, 1998) proposed successive quadratic programming method to solve the ORPD problem. The simulation results proved the efficiency of this method versus the linear programming method.

Yan et.al (Yan et al., 2006) and Granville (Granville, 1994) presented the primal dual interior points method - thereby succeeding in proposing a new model to optimize the allocation of reactive power by maintaining constant Hessian matrix during iterations, thus improving the total calculation time. Also succeeded in reducing the number of control variables, increased robustness and the possibility of applying the method on large networks. Over time, there have been many researches on reactive power dispatch optimization, with diverse purposes as:

- Increasing power system stability.

- Improve voltage profile.

Reduce network losses and thus increase economic efficiency in operation

To address this problem, several approaches have been proposed with varying degrees of efficiency and ease of implementation. These techniques fall generally into two classes:

- Classical techniques

- Techniques based on artificial intelligence (ai)

\subsection{Techniques based on AI}

Evolution is perhaps the most complex and most successful process of solving problems facing the species in a given situation. The main mechanism of change and adaptation in evolution is the natural selection. Charles Robert Darwin defines in his 
book, 'The Origin of Species' natural selection as "the principle that each slight variation is kept if it is useful."

The pioneering work of $\mathrm{JH}$ Holland in the 70s made an important contribution to the field of engineering and it established the bases for evolutionary computation. Later research in this area has experienced exponential growth, being initiated mostly by academic institutions. Although their benefits were superior to other methods of calculation, the difficulty to increase computational speed has led some experts to avoid their use. Important recent progress has been achieved due to the capability to produce powerful processors, widely available and at low prices.

Recently, techniques based on artificial intelligence have been developed, such as:

- Particle swarm optimization.

- Gravitational search algorithm.

- Genetic algorithms.

- Neural networks.

- Differential evolution algorithm.

These methods have the major advantage over classical methods, ie, that can solve complex problems, such as those of an area of nonlinear solutions, discontinuous and non-convex, without the need for an enhanced mathematical support. In addition, these techniques can solve problems containing discrete or binary variable, difficult to solve by other means.

Evolutionary computation techniques are optimization techniques based on the concept of evolving populations of individuals, thus improving their performance by probabilistic operators such as recombination and mutation. These individuals are evaluated and those who have higher performances have more chance of selection for training the next generation.

The generation represents a new population which is created in the algorithm at each iteration. It consists of the same number of individuals as previous generations. The individuals in each generation are better adapted to the environment represented by the fitness function. Thus, with the succession of generations, an evolution of individuals towards a global optimum function of adaptation is recorded (Eremia et al., 2006).

\section{The Proposed Method}

\subsection{Differential algorithm cycle}

Bhushan (Bhushan ,2013) proposed the usage of differential evolution algorithm to improve voltage profile and reduce losses on transmission lines by optimizing the allocation of reactive power.

DE uses a population of individuals and applies genetic operators (crossover, mutation, selection) for solving nonlinear problems, discontinuous and non-differentiable, unsolvable through classic methods listed above. (Booker \& Davis, 1987).

Stages addressed in the differential evolutionary algorithm are (Santosh \& Himmat, 2013) and (Bulac \& Eremia, 2006):

1. initialization

2. mutation 
3. recombination

4. selection

These steps are shown in the following chart:

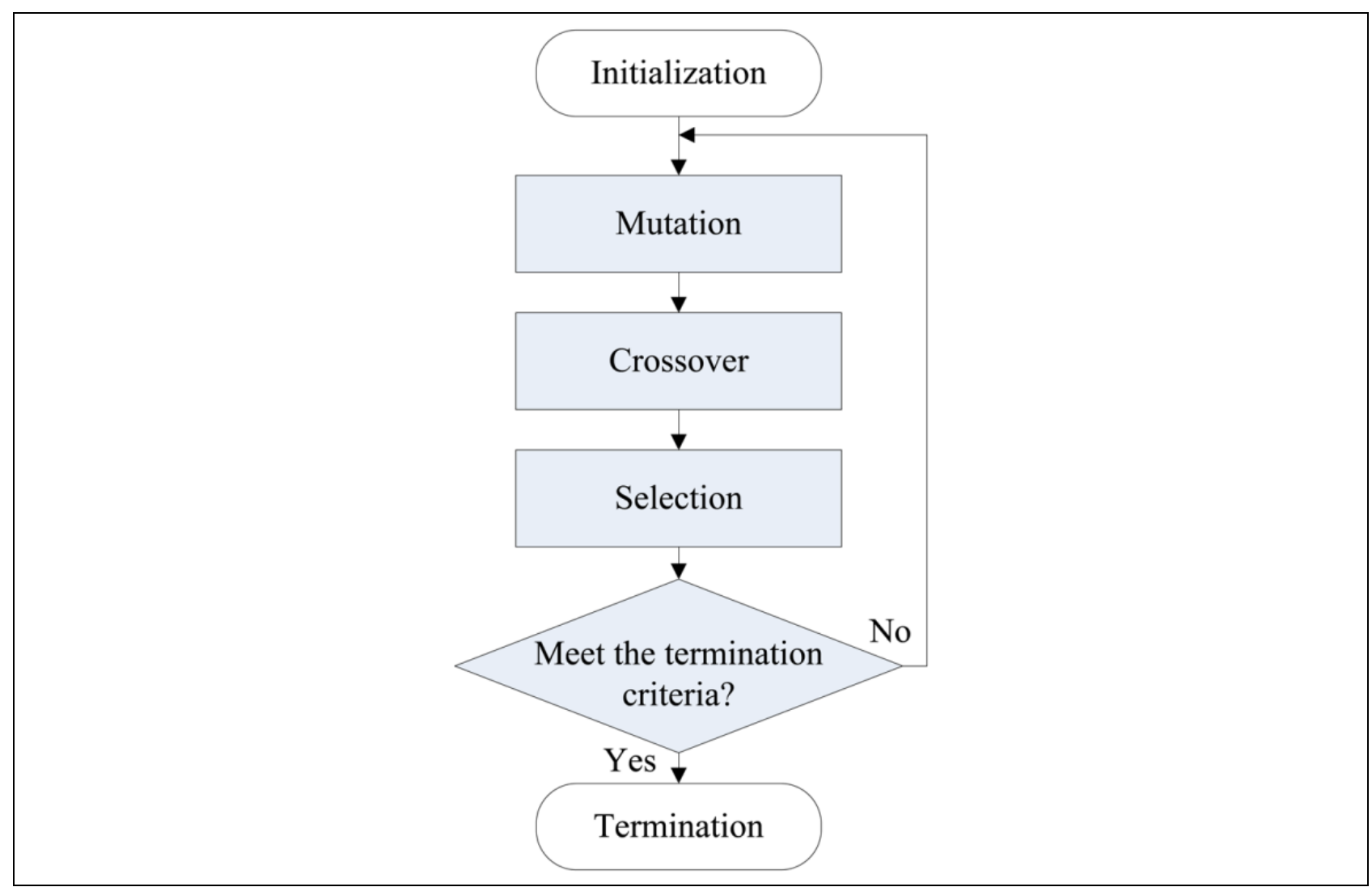

Fig. 1. Differential evolutionary algorithm cycle

\section{Initialization}

The initialization is the particle swarm optimization case, whose solution domain is defined by its boundaries, i.e. the minimum and maximum values of each control variable:

$$
x_{\min , k} \leq x_{i, k}(t) \leq x_{\max , k}, i=\overline{1, D_{p}}, k=\overline{1, n}
$$

where $D_{p}$ represents the size of the population and not the size of the search space. Therefore, population initialization is done according to:

$$
x_{i, k}(0)=x_{\min , k}+\operatorname{rand}() \times\left(x_{\max , k}-x_{\min , k}\right), i=\overline{1, D_{p}}, k=\overline{1, n}
$$

\section{Mutation}

The role of this operator is to keep diversity from one generation to another by changing one or more genes of an individual before being transferred to the next generation. This 
avoids creating too similar individuals, which can result in slowing the progress or stagnation. This type of operator introduces individuals in the population that could not be produced by other means.

Mutation operator is based, like the crossover operator, on probabilistic mechanisms, so each individual has a probability of mutation. The difference with other operators intervening in the value of this probability of mutation, which is of the order of 10-3 so motivated by natural processes where the mutation phenomena occur very rarely. It is the main operator used in the ADE. For each individual in the current population, called target vector, a mutant is generated (a new individual having new features) called donor vector. In this regard, three random vectors / individuals are selected which must be different from each other and the target vector. Then the donor vector is generated using the relationship:

$$
V_{i}(t)=X_{r_{1}}(t)+F\left(X_{r_{2}}(t)-X_{r_{3}}(t)\right)
$$

where $\mathrm{F}$ is a real scaling factor, which controls the amplitude of the difference vector.

\section{Recombination}

In order to concentrate the search in the most promising areas of the search space, the selection operator is used. The main disadvantage of this operator is that it cannot introduce new individuals in the population. To explore adjacent space areas that contain the current population, the most commonly used operators are: recombination and mutation.

Using the target vector and the donor vector, a new vector called test vector is generated. Basically there are two versions of the recombination / crossover operator.

The first version, called binomial recombination method, uses the following algorithm to select successor's components:

Step 1. Generate a random integer $r_{k} \in[1, n]$

Step 2. For each $k=\overline{1, n}$, it generates a random value $r_{1, k} \in[0,1]$ and set component $u_{i, k}(t)$ according to:

$$
u_{i, k}(t)=\left\{\begin{array}{c}
v_{i, k}(t), \text { if } r_{i, k} \leq C R \text { or } r_{k}=k \\
\text { else } x_{i, k}(t)
\end{array}\right.
$$

In $\mathrm{CR}$ is the probability that a component of the donor vector to be transmitted to the offspring. To ensure that at least one component of the offspring mutant vector will be transmitted, an additional condition has been introduced $r_{k}=k$ in relation.

The second method, known as the exponential method, is similar to the crossover with two cut points in the genetic algorithms. In this regard, the first point of cutting is selected randomly, and the second component is selected from the mutant vector. 


\section{Selection}

The selection has a decisive role for the search process in the solutions space. The selection operator aims to provide more opportunities for breeding the best performing individuals within a given population. It is therefore necessary to define a function for assessing individual performance to implement this operator.

Exploiting the best solutions is achieved by concentrating the search space solutions in the region corresponding to the maximum suitability, aiming to maximize performance.

Among the most popular methods of selection are: proportional selection (roulette wheel method), truncation selection (sequencing-based method), competitive selection (competition) and local selection.

The selection process is similar to the tournament type selection of the genetic algorithms. Thus, if the performance of the successor is better than the one of target vector, then the successor will replace the target vector in the new generation.

The search process continues until convergence criterion is fulfilled which usually consists of maximum number of generations.

\subsection{Differential evolutionary algorithm application}

The paper was discussed allocation of reactive power optimization problem in order to reduce active power losses. In this section we used the standard IEEE 30 network to demonstrate the effectiveness of the differential algorithm.

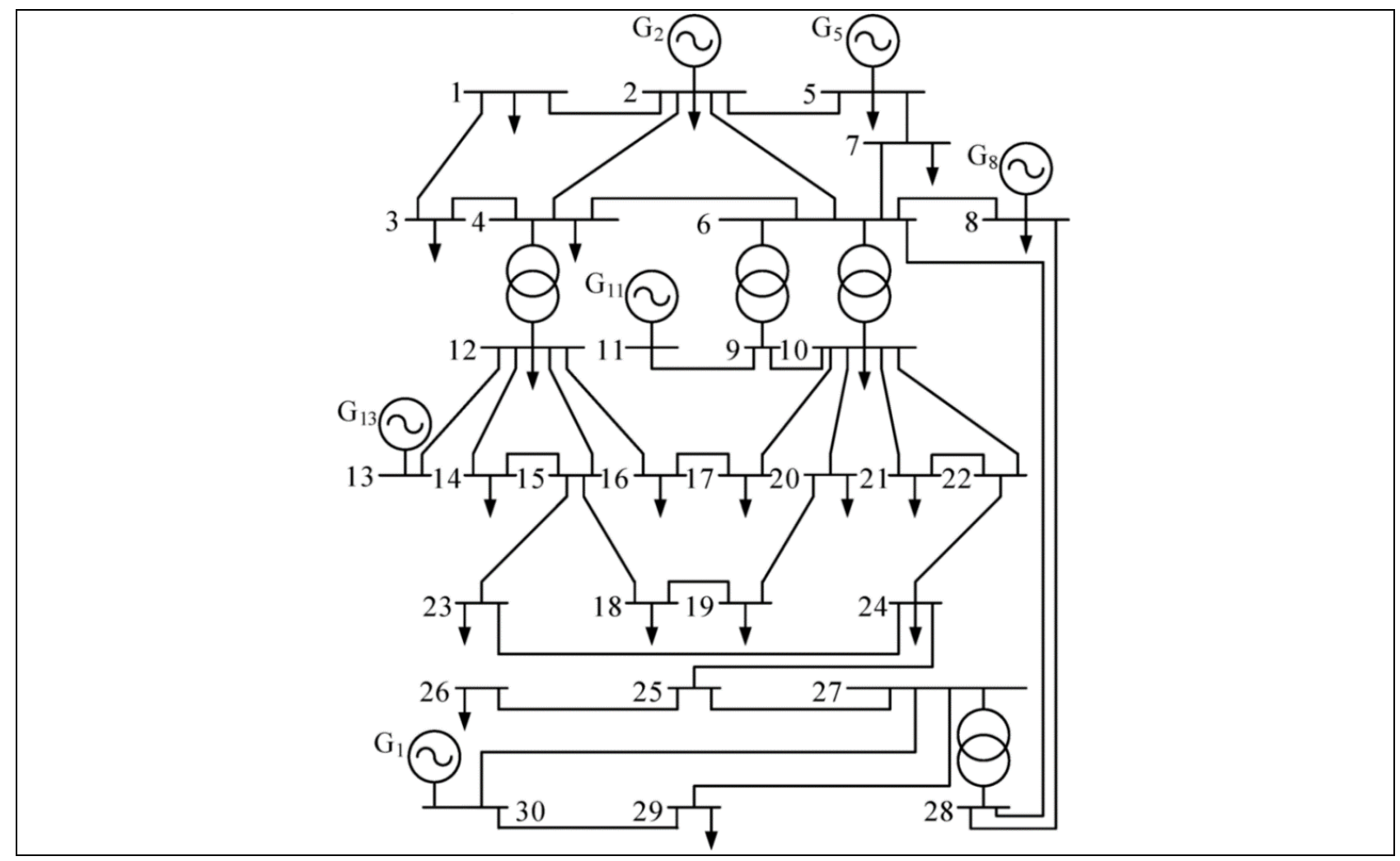

Fig. 2. IEEE 30 Bus Network

\section{a. Initial conditions}

- Voltage generators nodes is 1 [r.u]; 
- Transformers plots position is 0 ;

- Active power generated $P=2.8946_{G}$ [r.u];

- Active power consumption is $P_{C}=2.834$ [r.u];

- Reactive power is generated by $Q_{G}=1.3101$ [r.u];

- Reactive power is consumed $Q=1.2621_{C}$ [r.u];

- The active power losses are $D_{P}=0.0606$ [r.u];

\section{b. Differential evolutionary algorithm optimization solution.}

To use differential evolutionary algorithm in reactive power allocation optimization problem, the control variables of the transport network - voltage at PU nodes and position of the transformers tap - are arranged as individual elements of a population.

Table 1 presents the composition of these individuals.

\begin{tabular}{|l|l|l|l|l|l|l|l|l|l|l|}
\hline$p_{i}=$ & $U g 2$ & $U g 5$ & $U g 8$ & $U g 11$ & $U g 13$ & $U g 30$ & $t 6,9$ & $t 6,10$ & $t 4,12$ & $t 28,27$ \\
\hline
\end{tabular}

Tab.1. Composition of each individual population.

Initial parameters of the algorithm are:

- Population size for the selected algorithm is 50 individuals.

- The number of competitors is 20 .

- The maximum number of generations is 30 .

- Shift factor is 0.2 .

In the initial population of individuals, each element is initialized with a random value between the lower and upper limits of voltage / transformers plots. The performance of each individual is obtained by running the program for calculating the power flow based on the Newton-Raphson method.

\section{c. Simulation results}

Voltage of the network in the initial state is presented in the graph from figure 3.

As it can be seen, the system voltage is relatively low. In some situations, such as buses 1,26 and 29, the voltage is below the minimum threshold of 0.95 (r.u). 


\section{Bus Voltage}

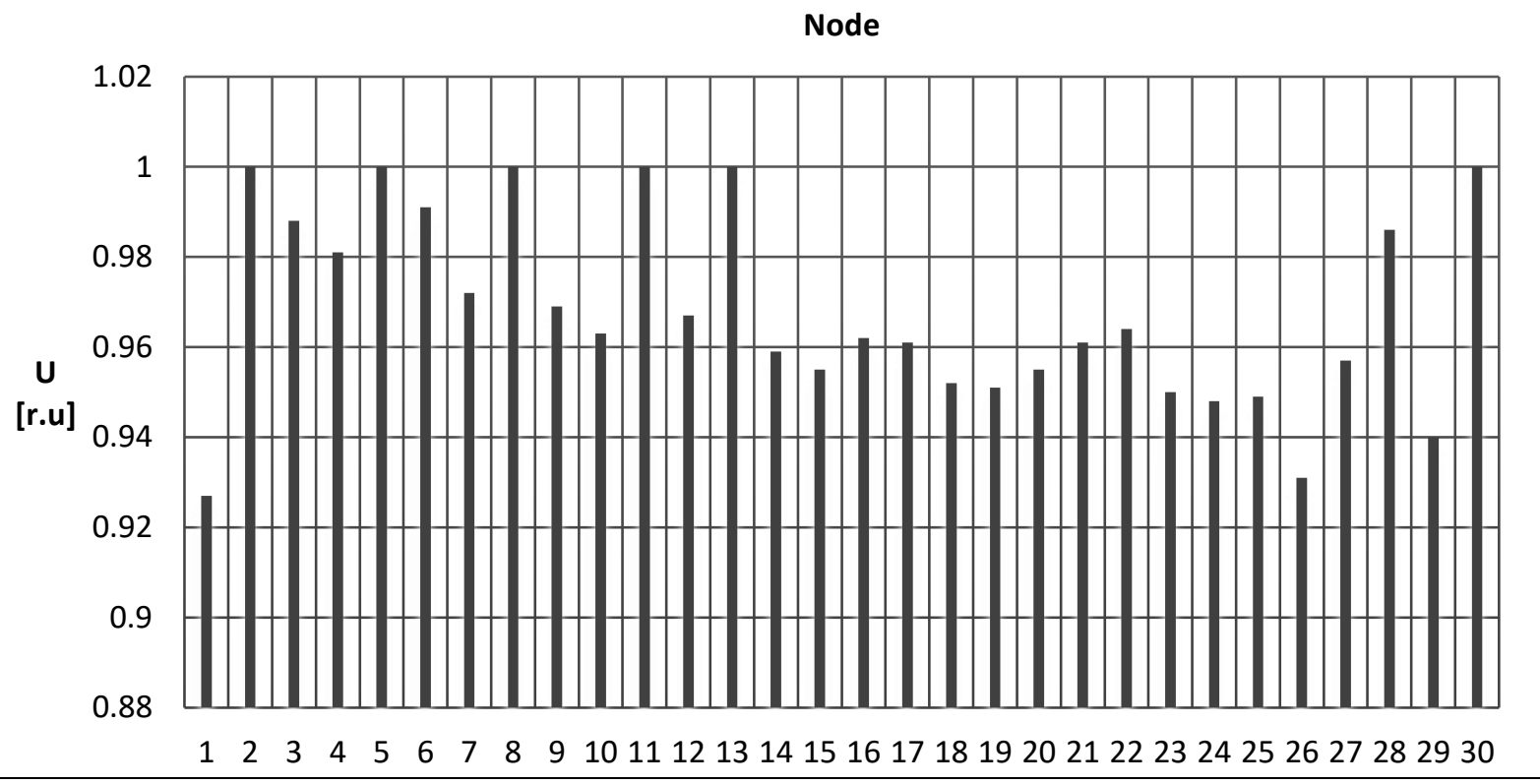

Fig. 3 Voltage of the network in the initial state

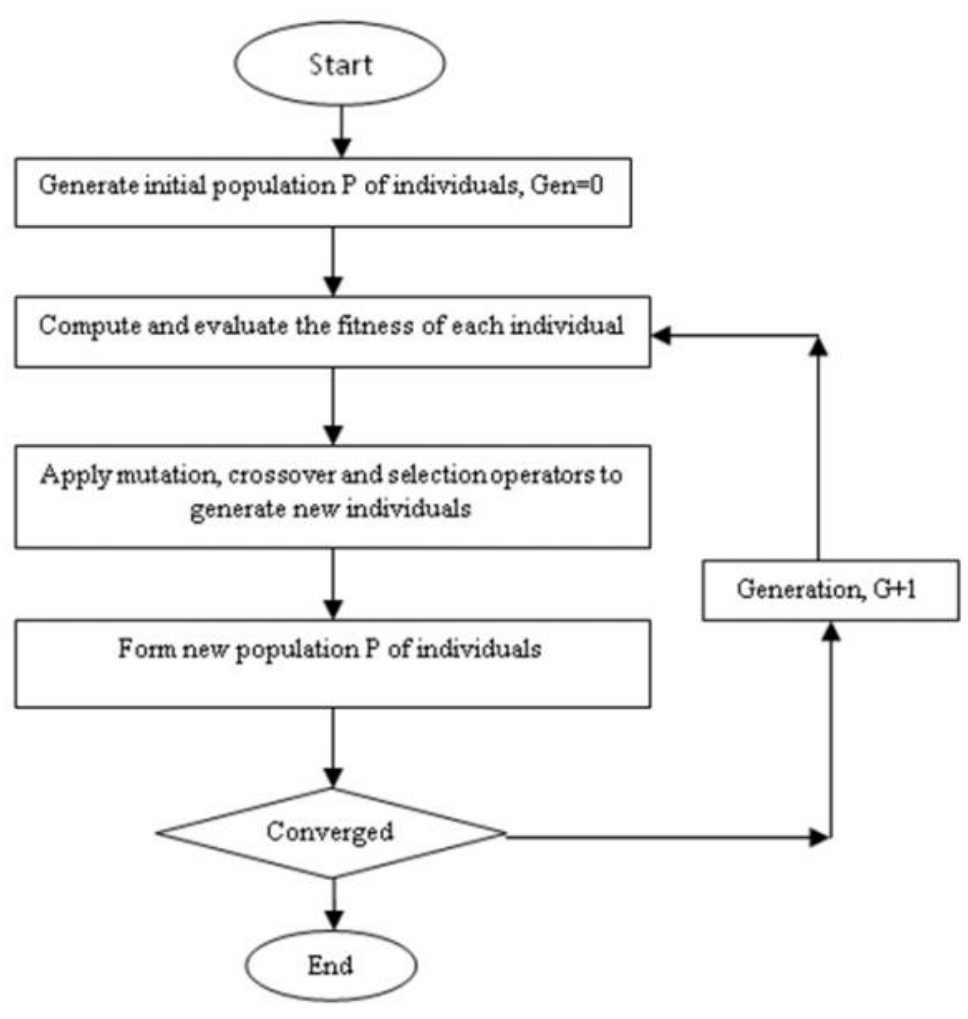

Fig. 4 Differential evolutionary algorithm cycle

This causes significant losses of active power and increased the instability of the network. 
The results were obtained using the MATLAB implementation of the algorithm mentioned above, the cycle of which is described in the figure 4.

After applying differential evolutionary algorithm were obtained following voltages at the nodes:

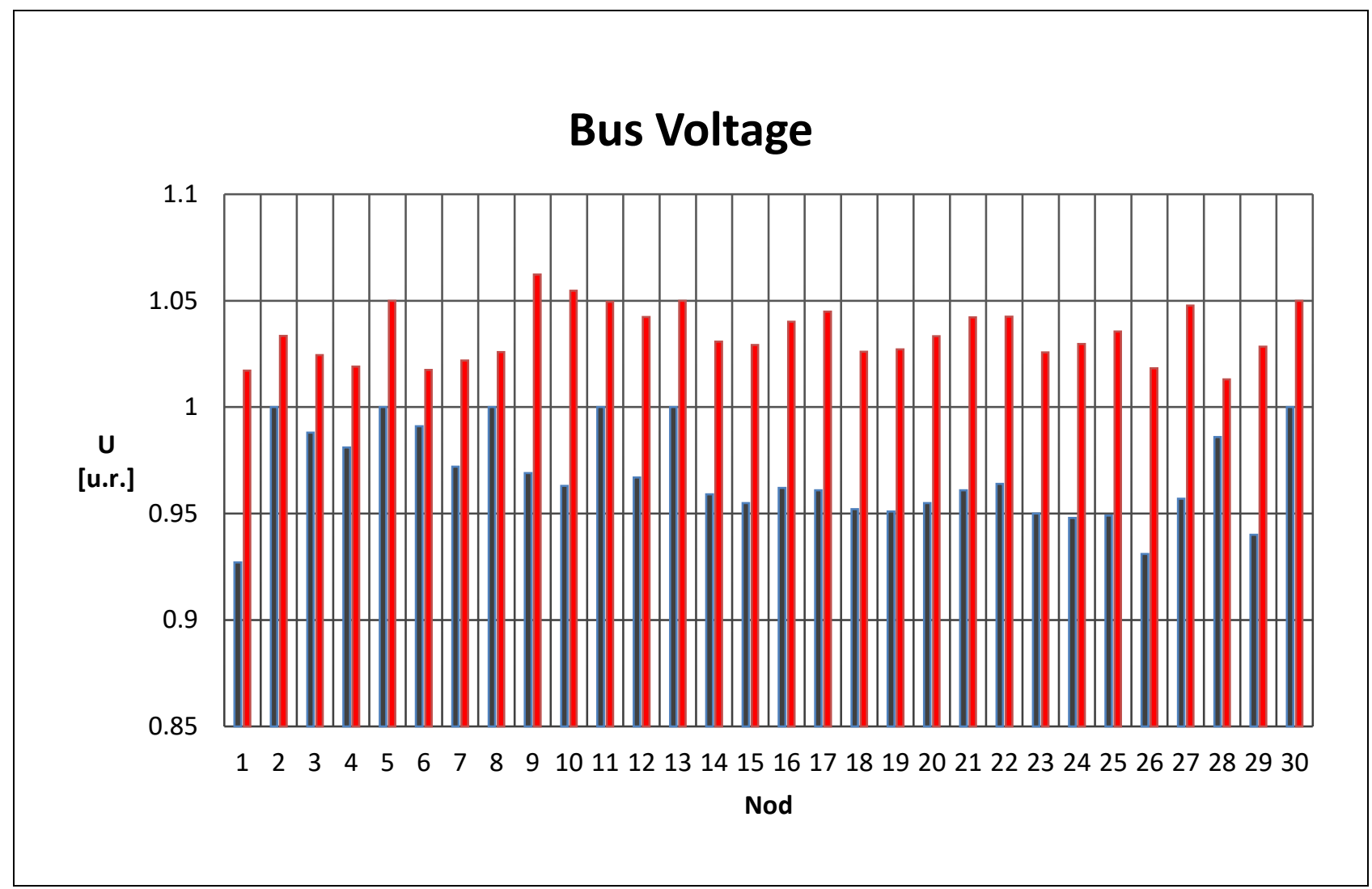

Fig. 5 Voltage of the network in the initial state (blue) versus voltage of the network state determined by DE ( red)

As can be seen, by using the DE algorithm, the voltage profile at the network buses is not violating the upper/lower limits.

The generated / consumed power inside the network are as follows:

- Active power generated $P=2.886_{G}$ [ur];

- Active power consumption is $P_{C}=2.834$ [ur];

- $Q$ reactive power generated is ${ }_{G}=1.241$ [ur];

- The active power losses are $D_{P}=0.0519742$ [ur];

Compared to the initial regime where losses were 0.0606 [r.u], there is a decrease in their value of $14.657 \%$.

This is illustrated in the chart from figure 6: 


\section{Active power losses}

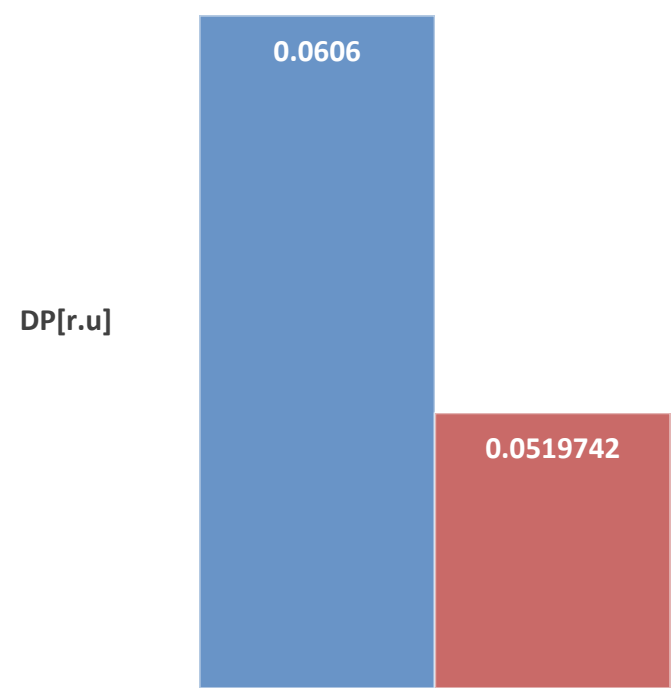

Fig.6. Comparison of the active power losses - Initial state (blue) versus the state determined using DE (red)

Using the characteristics of evolutionary computation procedures, final results may have slight variations. Thus it requires multiple simulations under the same conditions to evaluate the average performance of the program.

After ten consecutive simulations yielded the following percentages of optimization of the active power losses:

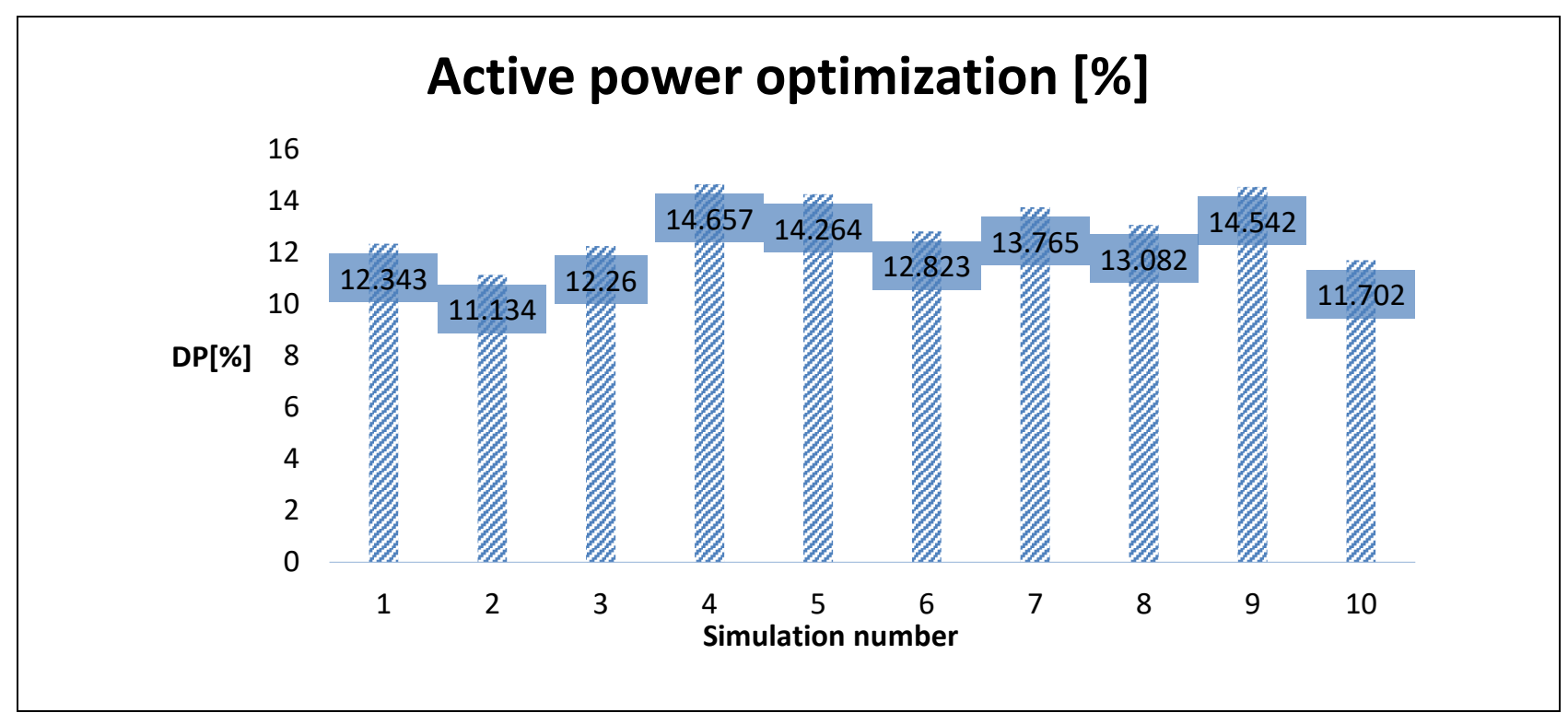

Fig. 7. Optimization of the active power losses as a resultfrom ten simulations

Mean active power optimization from these simulations is $13.057 \%$. After applying the algorithm in (Ionescu et al., 2010) achieved a maximum optimization of $13.354 \%$ with an average of $11.124 \%$. Thus, the results obtained are similar with those obtained in other papers. 


\section{Conclusions}

The results show that the differential evolutionary algorithm implemented was able to optimize the distribution of reactive power and, consequently, to reduce the active power loss in the system on the average by 13 percent up to a maximum of about 15 percent. It also managed to maintain the bus voltage within the limits.

Given these considerations, differential evolutionary algorithm could be successfully used for planning the dispatch of energy systems, being a powerful optimization tool, easy to use and implement, that can solve nonlinear problems in the presence of certain constraints.

\section{References}

Herbig, A. and Andersson, G., (1997). Control of power flows in transmission systems. International Power Engineering Conference, pg 431-435. Nanyang Technological University, Singapore

Bakare, G., A. (2001). Removal of Overloads and Voltage Problems in Electric Power Systems using Genetic Algorithm / Expert System Approaches, University of Duisburg , Department of electrical engineering

Kundur, P. (1993) Power System Stability and Control, Electric Power Research Institute

Grudinin, N. (1998). Reactive power optimization using successive quadratic programming method, IEEE Trans. Power Syst., vol. 13, no. 4, pp. 1219-1225,

Wei, Y.; Yu, J.; Yu D. C.; Bhattarai K.(2006) A new optimal reactive power flow model in rectangular form and its solution by predictor corrector primal dual interior point method, IEEE Trans. Power Syst., vol. 21, no. 1, pp, 61-67,.

Granville S.(1994). Optimal reactive dispatch through interior point methods, IEEE Trans. Power Syst., vol. 9, no. 1, pp. 136-146

Eremia, M.; Cârţină, Gh.; Petricică, D.; Bulac, A-I.; Bulac, C.; Triştiu, I.; Grigoraş, Gh. (2006). Tehnici de inteligenţă artificială în conducerea sistemelor electroenergetice, AGIR, Bucureşti

Bhushan ,W. (2013) "Optimization of reactive power for line loss reduction and voltage profile improvement using Differential Evolution Algorithm", International Journal of Enhanced Research in Science Technology \& Engineering, Vol. 2 Issue 12, pp. 29-34

Booker, L.; Davis, L. (1987) Genetic Algorithms and Stimulated Annealing, New York Pitman, , pp. 61-73

Santosh, K.; M., Himmat S. (2013). Reactive Power Optimization Using Differential Evolution Algorithm, IJETT

Bulac, C.;Eremia M. (2006). Dinamica sistemelor electroenergetice, Printech, Bucharest

Hrubina, K.; Katalinic, B.; Jadlovska, A.; Wessely, E.; Macurova, A.; Majercak, J. (2010). Modeling and Computing Methods for Solving Optimization Problems, DAAAM International Scientific Book, Chapter 42, p471

Ionescu, F.; Bulac, C.;Pisica, I.; Tristiu, I.; Toma, L. (2010). An Evolutionary Programming Application to Optimal Reactive Power Dispatch, U.P.B, Bucharest 